\title{
INFLUÊNCIA DO EXTRATO PIROLENHOSO NA CALDA DE PULVERIZAÇÃO SOBRE O TEOR FOLIAR DE NUTRIENTES EM LIMOEIRO 'CRAVO’'
}

\author{
MARCELO ZANETTI ${ }^{2}$, JAIRO OSVALDO CAZETTA ${ }^{3}$, DIRCEU DE MATTOS JÚNIOR ${ }^{4}$, \\ SÉRGIO ALVES DE CARVALHO ${ }^{4}$
}

\begin{abstract}
RESUMO - Com o objetivo de avaliar o efeito da presença do extrato pirolenhoso (EP) na calda de pulverização sobre o teor foliar de nutrientes de limoeiro 'Cravo' (Citrus limonia Osbeck), foi desenvolvido um experimento com seis tratamentos e quatro repetições, em blocos ao acaso, em ambiente protegido. Os tratamentos constituíram da pulverização das soluções: T0 = água; T1 = solução de micronutrientes sem EP; T2 = solução de micronutrientes + EP $\left(1 \mathrm{~cm}^{3} \mathrm{dm}^{-3}\right) ; \mathrm{T} 3=$ solução de micronutrientes $+\mathrm{EP}\left(2 \mathrm{~cm}^{3} \mathrm{dm}^{-3}\right) ; \mathrm{T} 4=$ solução de micronutrientes $+\mathrm{EP}\left(5 \mathrm{~cm}^{3} \mathrm{dm}{ }^{-3}\right) ; \mathrm{T} 5=\operatorname{solução}$ de micronutrientes $+\mathrm{EP}\left(10 \mathrm{~cm}^{3} \mathrm{dm}^{-3}\right)$. A solução de micronutrientes foi preparada com sulfatos de $\mathrm{Cu}, \mathrm{Fe}, \mathrm{Mn}, \mathrm{Zn}\left(250 \mathrm{mg} \mathrm{dm}^{-3} \mathrm{do}\right.$ elemento) e ácido bórico (42,5 mg dm $\mathrm{m}^{-3} \mathrm{de}$ B). As plantas foram cultivadas em tubetes cônicos de 0,280 $\mathrm{dm}^{3}$, com substrato sem a adição de micronutrientes na formulação. As soluções foram pulverizadas uma única vez, aos 140 dias após o plantio (DAP), momento em que as plantas apresentavam aproximadamente $20 \mathrm{~cm}$ de altura. Ao final do experimento (160 DAP), quantificaram-se a massa seca e os teores de macro e micronutrientes da parte aérea e sistema radicular. A presença do extrato pirolenhoso na solução de micronutrientes não interferiu na concentração foliar de B, Fe e Zn em mudas de limoeiro 'Cravo'. Entretanto, na concentração de $10 \mathrm{~cm}^{3} \mathrm{dm}^{-3}$, aumentou a concentração foliar de Cu e Mn. Observou-se também que as plantas pulverizadas com soluções contendo EP $\left(1\right.$ a $\left.10 \mathrm{~cm}^{3} \mathrm{dm}^{-3}\right)+$ micronutrientes apresentaram menor teor de Fe e maior teor de Ca no sistema radicular.
\end{abstract}

Termos para indexação: Citrus, propagação, pulverização foliar, produção de mudas, micronutrientes.

\section{EFFECT OF PYROLIGNEOUS ACID IN THE SPRAYMG SOLUTIONS ON FOLIAR NUTRIENTS CONTENT OF 'RANGPUR' LIME}

\begin{abstract}
This research studied the effect of pyroligneous acid (PA) presence in the micronutrient solution sprayed on leaves on the foliar nutrient content of 'Rangpur lime' (Citrus limonia Osbeck0) seedlings, under screen house. An experiment in a randomized complete block design with six treatments and four replicates was set up. Treatments consisted of leaf spraying with the following solutions: $\mathrm{T} 0=\mathrm{water}$; $\mathrm{T} 1=\mathrm{micronutrients}$ solution without PA; T2 = micronutrients solution $+\mathrm{PA}\left(1 \mathrm{~cm}^{3} \mathrm{dm}^{-3}\right) ; \mathrm{T} 3=$ micronutrients solution $+\mathrm{PA}\left(2 \mathrm{~cm}^{3} \mathrm{dm}^{-3}\right)$; $\mathrm{T} 4=$ micronutrients solution $+\mathrm{PA}$ $\left(5 \mathrm{~cm}^{3} \mathrm{dm}^{-3}\right)$; T5 = micronutrients solution $+\mathrm{PA}\left(10 \mathrm{~cm}^{3} \mathrm{dm}^{-3}\right)$. The micronutrient solutions were prepared by using sulphates of $\mathrm{Cu}, \mathrm{Fe}, \mathrm{Mn}$ and $\mathrm{Zn}(250$ $\mathrm{mg} \mathrm{dm}{ }^{-3}$ of the elements), and boric acid ( $42.5 \mathrm{mg} \mathrm{dm}^{-3}$ of B). The experiment was set up on $0.280 \mathrm{dm}^{3}$ conical containers, in growing media with no addition of micronutrients. The solutions were sprayed once, at 140 days after planting (DAP), when the plants were about $20 \mathrm{~cm}$ high. At the end of the experiment (160 DAP), dry mass of plants and concentrations of nutrients of aerial part and root system were evaluated. The presence of pyroligneous acid in the spraying solution did not affect B, Fe and $\mathrm{Zn}$ concentration in the leaves. However, increased $\mathrm{Cu}$ and $\mathrm{Mn}$ concentration for treatments containing PA at $10 \mathrm{~cm}^{3} \mathrm{dm}^{-3}$ in the micronutrients solution. It was also observed that plants sprayed with solutions of PA (1 to $\left.10 \mathrm{~cm}^{3} \mathrm{dm}^{-3}\right)$ + micronutrients showed lower $\mathrm{Fe}$ and higher $\mathrm{Ca}$ concentration on the root system.
\end{abstract}

Index terms: Citrus, propagation, leaf spray, nursery tree production, micronutrients.

\section{INTRODUÇÃo}

A cultura dos citros tem grande importância no Brasil, visto que o País é o maior produtor de laranja do mundo. A qualidade das mudas cítricas é um dos fatores que determinam o sucesso dos cultivos comerciais, já que a planta somente revelará seu potencial máximo de produtividade seis a oito anos após o plantio.

As mudas cítricas devem ser produzidas em vasos ou sacolas plásticas, em sistema protegido, com o uso de substrato isento de patógenos e adequados a este tipo de produção. Neste processo de produção de mudas, nos quais altas taxas de crescimento das plantas são obtidas em curto espaço de tempo, torna-se essencial a suplementação mineral, via adubação.

Dentre as alternativas no manejo de adubação, isoladamente ou associados, estão os adubos de liberação controlada, o fornecimento de nutrientes ao substrato via água de irrigação e a adubação foliar. A escolha do manejo mais apropriado, de formulações e de freqüências de aplicação tem sido realizada com base na experiência individual dos viveiristas, havendo carência na literatura de recomendações específicas para este novo sistema de produção de mudas.

De acordo com Boaretto \& Rosolem (1989), a adubação foliar tem como objetivo complementar a nutrição realizada via solo ou substrato e suprir, em quantidade e qualidade, as necessidades nutricionais das plantas, além de possibilitar a correção mais rápida de determinadas deficiências. A eficiência da adubação foliar pode ser avaliada por diferentes métodos, sendo o mais comum, e prático, o método indireto, no qual se realiza a pulverização de nutrientes sobre as folhas e, decorridos 20 a 60 dias para a absorção dos mesmos, quantificase a alteração da composição química da planta. Este método, porém, não permite quantificar a porcentagem da quantidade aplicada que foi absorvida pelas folhas nem quantificar a translocação do nutriente para outros órgãos (Boaretto et al., 2003).

A velocidade de absorção dos elementos varia com a planta e com o elemento. De acordo com Malavolta et al. (1997), 50\% do Fe e Mn, aplicados através da pulverização foliar, são absorvidos após um ou dois dias, e o Zn, após 2 a 24 horas. Todavia, Boaretto et al. (2003), utilizando a técnica de auto-radiografia em plantas cítricas, relatam que a velocidade de absorção é dependente da fonte empregada e da quantidade aplicada.

Trabalhos desenvolvidos com plantas adultas no campo têm demonstrado que a pulverização foliar de solução contendo micronutrientes aumentou significativamente seus teores nas folhas de citros colhidas 20 ou 60 dias após a aplicação (Caetano, 1982; Tiritan, 1996; Santos et al., 1999).

Boaretto et al. (2003) confirmaram que duas aplicações anuais de micronutrientes ( $770 \mathrm{mg} \mathrm{dm}^{-3} \mathrm{Zn}, 625 \mathrm{mg} \mathrm{dm}^{-3} \mathrm{Mn}$ e $170 \mathrm{mg} \mathrm{dm}^{-3} \mathrm{~B}$ ),

\footnotetext{
${ }^{1}$ (Trabalho 094/2004). Recebido em: 21/07/2004. Aceito para publicação: 17/12/2004. Apoio: BIOCARBO. Parte da Dissertação de Mestrado em Agronomia Produção Vegetal / FCAV - UNESP - Jaboticabal-SP.

${ }^{2}$ Mestrado em Agronomia (Produção Vegetal) da UNESP - Universidade Estadual Paulista - Jaboticabal-SP. zanettimarcelo@ yahoo.com.br

${ }^{3}$ Professor da UNESP- Universidade Estadual Paulista - Jaboticabal-SP. cazetta@fcav.unesp.br

${ }^{4}$ Centro APTA Citros “Sylvio Moreira” - CAPTACMS/IAC/SAA, Cordeirópolis-SP.
} 
conforme indicações do Grupo Paulista de Adubação e Calagem para Citros - GPACC (Quaggio et al., 1997), têm sido eficientes para elevar os teores foliares de $\mathrm{Zn}$, Mn e B, mas é insuficiente para alterar o teor nas folhas novas das laranjeiras que nascem após a pulverização, devido à baixa translocação dos mesmos para outros órgãos da planta.

A eficiência da adubação foliar pode ser influenciada por diversos fatores, entre as quais: o ambiente (luz, temperatura e umidade); fatores intrínsecos à própria planta (superfície foliar, cutícula e idade da folha), e a solução (composição, $\mathrm{pH}$, carga, forma e concentração dos nutrientes) (Pedras et al., 1989; Malavolta et al., 1997).

Para que o nutriente, colocado na superfície da folha, entre no espaço intracelular, é necessário que os mesmos atravessem a cutícula, a parede celular epidérmica e o plasmalema. A passagem do íon através da membrana pode ocorrer de maneira passiva, através de um gradiente de concentração, ou ativa, com o gasto de energia (Boaretto et al., 2003).

Há bastante tempo, vem se ressaltando a importância da composição da solução usada como veículo dos nutrientes, principalmente a mistura de outros solutos como agentes protetores e surfatantes (Camargo \& Silva, 1975). Recentemente, tem sido sugerida a mistura de extrato pirolenhoso (EP) nas soluções pulverizadas nas plantas, visando a uma série de benefícios (Miyasaka et al., 2001), mas ainda não se têm informações consistentes sobre o seu efeito na absorção foliar de micronutrientes.

O extrato pirolenhoso (EP) é um líquido obtido através da condensação da fumaça produzida durante o processo de carbonização da madeira. Este líquido é constituído de 800 a $900 \mathrm{~cm}^{3} \mathrm{dm}^{-3}$ de água, e o restante contempla uma série de diferentes componentes químicos, predominando, quantitativamente, o ácido acético, o metanol, a acetona e os fenóis.

O EP vem sendo pesquisado principalmente no Japão e têm sido relatados resultados promissores como "fertilizante orgânico" em arroz (Oryza sativa L.) (Tsuzuki et al., 2000), sorgo (Sorghum bicolor L.) (Esechie et al., 1998), batata-doce (Ipomoea batatas L.) (Shibayama et al., 1998). Com base em resultados de pesquisa, como os acima citados, produtores, principalmente os ligados à chamada agricultura natural, estão utilizando o extrato pirolenhoso na produção hortifrutigranjeira, como insumo agrícola natural (Biocarbo Indústria e Comércio, 2003).

Há indícios de que as características físicas e químicas, especialmente o conteúdo de substâncias com potencial quelatizante do extrato pirolenhoso, poderiam potencializar a eficiência de produtos fitossanitários e a absorção de nutrientes em pulverizações foliares. De acordo com Ponchio \& Ballio (1988), substâncias quelatizantes são aquelas que impedem reações indesejáveis de um micronutriente metálico em solução; pela formação de uma estrutura ao seu redor que os modifica quimicamente, através de ligações covalentes coordenadas com ligantes, formando uma estrutura anelar heterocíclica.

De acordo com Wallace (1956), substâncias naturais, como ácido cítrico, ácido húmico, aminoácidos e lignossulfonados de amônio, subprodutos da indústria de polpa de madeira, entre outros, podem ser utilizados como quelantes naturais, principalmente na pulverização foliar.
No que se refere ao extrato pirolenhoso, existe carência de informações experimentais, especialmente relacionadas com a avaliação da real capacidade quelante das substâncias presentes no extrato pirolenhoso e, menos ainda, com a aplicação deste insumo na cultura dos citros em condições brasileiras.

Dentro desse contexto, este trabalho teve como objetivo avaliar o efeito da presença de extrato pirolenhoso na solução de micronutrientes pulverizada na parte aérea, sobre o teor de nutrientes de mudas de limoeiro 'Cravo' (C. limonia Osbeck), cultivadas em ambiente protegido, como um primeiro estudo para balizar a utilização do EP ou o desenvolvimento de estudos mais específicos.

\section{MATERIALEMÉTODOS}

O trabalho foi desenvolvido entre fevereiro e agosto de 2003, no viveiro comercial da Citrograff - Mudas, em Rio Claro-SP. O viveiro é coberto por filme plástico transparente e com telado antiafídeos nas laterais, de acordo as exigências da legislação para a produção de mudas de citros no Estado de São Paulo (Carvalho, 2003).

Foram utilizadas sementes de limoeiro Cravo $(C$. limonia Osbeck cv Limeira) retiradas de frutos colhidos de plantas-matrizes registradas. As sementes foram semeadas em 26-02-2003 em tubetes de plástico, em formato de cone e volume de $0,280 \mathrm{dm}^{3}$. Os tubetes foram alocados em bandejas plásticas perfuradas, contendo 54 células, fixadas sobre armação de ferro suspensa. Utilizou-se substrato comercial Plantmax ${ }^{\circledR}$ (Eucatex Ltda: Paulínia/SP) sem adição de micronutrientes, mas com a adição de fertilizante de disponibilidade lenta (Osmocote ${ }^{\circledR}$ N-P-K, 18-5-9: Scotts/ USA) misturado ao substrato, na proporção de $4 \mathrm{~g} \mathrm{dm}^{-3}$.

Para a caracterização do meio de cultivo utilizado no experimento, o substrato foi analisado no Laboratório do Centro de Pesquisa e Desenvolvimento de Solos e Recursos Ambientais, do Instituto Agronômico de Campinas-SP, pelo método da extração aquosa 1:1,5 (v/ v) descrito por Sonneveld et al. (1974) e apresentou as seguintes características químicas: $\mathrm{N}_{-} \mathrm{NH}_{3}=19,08 ; \mathrm{N}^{-N_{3}}{ }^{-}=6,07 ; \mathrm{P}=1,8 ; \mathrm{K}=95,3$; $\mathrm{Ca}=69,9 ; \mathrm{Mg}=33,6 ; \mathrm{S}=138,6 ; \mathrm{B}=0,06 ; \mathrm{Cu}=0,03 ; \mathrm{Fe}=0,09 ; \mathrm{Mn}=0,55$; $\mathrm{Zn}=0,04$ (com todos os valores expressos em $\left.\mathrm{mg} \mathrm{dm}^{-3}\right) ; \mathrm{pH}=5,0$; condutividade elétrica $=1,20 \mathrm{dS} \mathrm{m}^{-1}$, e umidade $=46 \%$.

$\mathrm{O}$ experimento foi conduzido com seis tratamentos e quatro repetições, em blocos ao acaso. Cada parcela foi composta por 24 plantas, sendo as 12 centrais compondo a área útil. Os tratamentos foram constituídos por pulverização das soluções: $\mathrm{T} 0$ = água; $\mathrm{T} 1$ = Solução de micronutrientes sem EP; T2 = Solução de micronutrientes + EP $\left(1 \mathrm{~cm}^{3}\right.$ $\left.\mathrm{dm}^{-3}\right) ; \mathrm{T} 3=$ Solução de micronutrientes $+\mathrm{EP}\left(2 \mathrm{~cm}^{3} \mathrm{dm}^{-3}\right) ; \mathrm{T} 4=$ Solução de micronutrientes $+\operatorname{EP}\left(5 \mathrm{~cm}^{3} \mathrm{dm}^{-3}\right) ; \mathrm{T} 5=$ Solução de micronutrientes + EP $\left(10 \mathrm{~cm}^{3} \mathrm{dm}^{-3}\right)$. A solução de micronutrientes foi preparada com os sulfatos de $\mathrm{Cu}, \mathrm{Fe}, \mathrm{Mn}, \mathrm{Zn}$ ( $250 \mathrm{mg} \mathrm{dm}^{-3}$ do elemento) e ácido bórico $\left(42,5 \mathrm{mg} \mathrm{dm}^{-3} \mathrm{de} B\right)$. Levando em conta que os sais utilizados não eram padrões-primários, para a perfeita caracterização, as soluções foram analisadas em laboratório, e os resultados encontram-se na Tabela1.

TABELA 1 - Teores totais de micronutrientes, pH e condutividade elétrica (EC) das diferentes soluções utilizadas no experimento para a determinação da influência do extrato pirolenhoso sobre o teor foliar de nutrientes, em limoeiro 'Cravo' cultivado em tubetes. Jaboticabal, UNESP, 2003.

\begin{tabular}{|c|c|c|c|c|c|c|c|c|}
\hline & \multirow{2}{*}{ TRATAMENTOS } & B & $\mathrm{Cu}$ & $\mathrm{Fe}$ & $\mathrm{Mn}$ & $\mathrm{Zn}$ & $\mathrm{PH}$ & $\mathrm{EC}$ \\
\hline & & \multicolumn{5}{|c|}{$\mathrm{mg} \mathrm{dm}{ }^{-3}$} & & $\mathrm{dS} \mathrm{m}^{-}$ \\
\hline T0 & Testemunha (água destilada) & 0 & 0 & 0 & 0 & 0 & 6,50 & 0,01 \\
\hline $\mathrm{T} 1$ & Micro + água destilada & 42 & 265 & 257 & 251 & 254 & 3,28 & 3,01 \\
\hline $\mathrm{T} 2$ & Micro + EP $1 \mathrm{~cm}^{3} \mathrm{dm}^{-3}$ & 45 & 270 & 252 & 252 & 252 & 3,25 & 3,03 \\
\hline $\mathrm{T} 3$ & Micro + EP $2 \mathrm{~cm}^{3} \mathrm{dm}^{-3}$ & 43 & 270 & 251 & 254 & 252 & 3,22 & 3,05 \\
\hline $\mathrm{T} 4$ & Micro + EP $5 \mathrm{~cm}^{3} \mathrm{dm}^{-3}$ & 42 & 267 & 251 & 251 & 252 & 3,15 & 3,12 \\
\hline T5 & Micro + EP $10 \mathrm{~cm}^{3} \mathrm{dm}^{-3}$ & 44 & 267 & 259 & 252 & 252 & 3,07 & 3,18 \\
\hline- & Média (exceto testemunha) & 43 & 268 & 254 & 252 & 252 & 3,19 & 3,08 \\
\hline
\end{tabular}


TABELA 2 - Teores médios dos nutrientes e desvios-padrão encontrados para a parte aérea e o sistema radicular de mudas de limoeiro 'Cravo', aos 160 DAP. Jaboticabal, UNESP, 2003.

\begin{tabular}{|c|c|c|c|c|c|c|c|c|c|c|c|}
\hline \multirow[t]{2}{*}{ Nutriente } & $\mathrm{N}$ & $\mathrm{P}$ & $\mathrm{K}$ & $\mathrm{Ca}$ & $\mathrm{Mg}$ & $\mathrm{S}$ & $\mathrm{B}$ & $\mathrm{Cu}$ & $\mathrm{Fe}$ & $\mathrm{Mn}$ & $\mathrm{Zn}$ \\
\hline & \multicolumn{6}{|c|}{$\mathrm{g} \mathrm{kg}^{-1}$} & \multicolumn{5}{|c|}{$\mathrm{mg} \mathrm{kg}^{-1}$} \\
\hline Parte aérea & 18,3 & 1,3 & 20,4 & 12,1 & 1,9 & 1,3 & 109,7 & 3,95 & 97,9 & 16,4 & 15,4 \\
\hline Desvio-Padrão & 0,9 & 0,1 & 2,2 & 1,1 & 0,1 & 0,1 & 5,7 & 1,2 & 6,2 & 1,1 & 1,1 \\
\hline Sistema radicular & 13,2 & 0,9 & 14,2 & 4,9 & 1,9 & 1,3 & 39,2 & 3,05 & 416,0 & 73,3 & 17,2 \\
\hline Desvio-Padrão & 0,7 & 0,1 & 1,5 & 0,3 & 0,1 & 0,1 & 2,8 & 1,4 & 57,1 & 10,8 & 8,6 \\
\hline
\end{tabular}

Como não foi encontrada, na literatura consultada, indicação para adubação foliar em mudas cítricas, partiu-se da recomendação para planta adulta descrita pelo Grupo Paulista de Adubação e Calagem para Citros - GPACC (Quaggio et al., 1997). Entretanto, aquela solução induziu fortes sintomas de fitotoxidez. Por isso, foram testadas subdoses até não mais se verificar sintomas de fitotoxidez, resultando, então, na solução acima descrita e cujas características estão na Tabela 1. O extrato pirolenhoso destilado foi fornecido pela Biocarbo Indústria e Comércio Ltda: Nova Lima-MG e, de acordo com análise realizada no laboratório da UNESP - Jaboticabal-SP, possui apenas traços de N, P, K, Ca, Mg, S e B, mas contém os seguintes micronutrientes: $\mathrm{Cu}\left(20,8 \mathrm{mg} \mathrm{dm}^{-3}\right) ; \mathrm{Fe}(3,8$ $\left.\mathrm{mg} \mathrm{dm}{ }^{-3}\right) ; \operatorname{Mn}\left(0,04 \mathrm{mg} \mathrm{dm}^{-3}\right)$, e Zn $\left(0,2 \mathrm{mg} \mathrm{dm}^{-3}\right)$.

As soluções foram pulverizadas uma única vez, aos 140 DAP (dias após o plantio), quando as plantas apresentavam aproximadamente $20 \mathrm{~cm}$ de altura. Utilizou-se um pulverizador costal, com bico do tipo cone vazio, com vazão de $0,5 \mathrm{dm}^{3}$ minuto ${ }^{-1} \mathrm{e}$ válvula de pressão constante de $1 \mathrm{kgf} \mathrm{cm}^{-3}$. A calda foi aplicada em quantidade suficiente para molhar completamente as plantas até o ponto de escorrimento, protegendo-se o substrato com placas de isopor para evitar que a solução entrasse em contato com o substrato e, assim, garantir que a solução atingisse apenas a parte aérea. Após a aplicação dos tratamentos, a irrigação foi realizada rente à superfície do substrato, de maneira que não houvesse a lavagem das folhas, para evitar que a solução depositada no limbo foliar entrasse em contato com o substrato e fosse absorvida pelas raízes.

Aos 160 DAP (20 dias após a aplicação dos tratamentos), foram realizadas avaliações da produção de massa seca $\left(\right.$ g planta $\left.^{-1}\right)$ e determinação dos teores de macro e micronutrientes da parte aérea e raiz. Para a determinação dos teores de nutrientes, as amostras da parte aérea e raízes foram lavadas, em seqüência, com: água corrente, detergente $\left(1 \mathrm{~cm}^{3} \mathrm{dm}^{-3}\right)$, água destilada, $\mathrm{HCl}\left(30 \mathrm{~cm}^{3} \mathrm{dm}^{-3}\right)$ e água destilada. Em seguida, as amostras foram secadas em estufa com circulação forçada de ar, com temperatura variando de $65 \mathrm{a} 70^{\circ} \mathrm{C}$, até atingirem peso constante. Depois foram moídas em moinho tipo Willey e submetidas à determinação dos teores totais de $\mathrm{N}$ pelo método de Kjeldahl; P por espectrofotometria; $\mathrm{S}$ por turbidimetria, $\mathrm{K}, \mathrm{Ca}, \mathrm{Mg}, \mathrm{Cu}, \mathrm{Fe}, \mathrm{Mn}$ e $\mathrm{Zn}$ através de digestão nítrico-perclórica e posterior quantificação por espectrofotometria de absorção atômica, além do teor de B pelo método da azometina-H, de acordo com metodologia descrita por Tedesco et al. (1995).

Os resultados foram submetidos à análise de variância, pelo Teste F, e as médias comparadas pelo teste de Tukey $(\mathrm{p}<0,05)$.

\section{RESULTADOSE DISCUSSÃO}

Os resultados obtidos para a produção de massa seca, tanto da parte aérea (média de 2,25 $\mathrm{g}_{\text {planta }}{ }^{-1}$ ) como do sistema radicular (média de $1,21 \mathrm{~g} \mathrm{planta}^{-1}$ ), não apresentaram diferenças significativas para os tratamentos, visto que as pulverizações foram realizadas 20 dias antes das determinações de massa seca. Face ao exposto, os resultados estatísticos dos dados de absorção de nutrientes por planta ou por unidade de matéria seca foram semelhantes e, portanto, optou-se por expressar e discutir os resultados com base nos teores foliares.

Os teores médios de nutrientes, assim como os desvios-padrão dos dados encontrados para a parte aérea e o sistema radicular, estão apresentados na Tabela 2. Deve-se ressaltar que os teores de macronutrientes e micronutrientes das plantas, apresentados no presente artigo, não devem ser confrontados com os parâmetros contidos nas tabelas de interpretação dos teores foliares para se avaliar o estado nutricional das plantas utilizadas neste experimento. Tal afirmativa se baseia no fato de que as tabelas de interpretação dos teores sugeridas pelo Grupo Paulista de Adubação e Calagem para Citros - GPACC (Quaggio et al., 1997) correspondem à terceira ou quarta folha a partir do fruto, gerada na primavera, com aproximadamente seis meses de idade, em ramos com frutos de $2 \mathrm{a} 4 \mathrm{~cm}$ de diâmetro. Como não existem dados referentes a porta-enxertos cultivados em substrato e sob condição de ambiente protegido, no presente estudo, analisou-se toda a parte aérea (folha + caule) das plantas.

Com relação aos teores de nutrientes no sistema radicular, foram constatadas diferenças significativas para $\mathrm{Ca}$ e Fe (Tabela 3).

O extrato pirolenhoso pulverizado na parte aérea, independentemente da concentração, provocou redução em cerca de $38 \%$ nos teores de Fe e aumento de $25 \%$ do $\mathrm{Ca}$ (Tabela 3 ).

Os teores de $\mathrm{Fe}$ no sistema radicular do limoeiro 'Cravo' foram muito superiores aos demais micronutrientes (Tabela 2) e, ainda, maiores nos tratamentos em que não se aplicou o EP (Tabela 3), o que pode ter interferido de forma negativa na absorção do cálcio nos tratamentostestemunha e no tratamento contendo apenas micronutrientes, pois, de acordo com Kabata-pendias \& Pendias citados por Dechen et al. (1991), o Ca apresenta antagonismo com os micronutrientes B, Cu, Fe, $\mathrm{Zn}$ e Mn.

Com relação aos teores de nutrientes na parte aérea, observaram-se diferenças significativas para B, Cu, Fe, Mn e Zn, cujos resultados se encontram na Tabela 4.

Os teores de $\mathrm{B}$ das plantas dos diferentes tratamentos não diferiram daquele verificado nas plantas do tratamento usado como testemunha (T0), uma vez que as deste apresentaram valores intermediários. Entretanto, o teor de B nas plantas foi diminuindo, consistentemente, à medida que se aumentou o nível de EP na solução, de modo que o teor nas plantas pulverizadas com EP $1 \mathrm{~cm}^{3} \mathrm{dm}^{-3}$ foi significativamente maior que nos tratamentos com $10 \mathrm{~cm}^{3} \mathrm{dm}^{-3}$.

A aplicação de B através da adubação foliar em plantas cítricas vem sendo bastante discutida. Boaretto et al. (2003) afirmam que o B é rapidamente absorvido pelas folhas, porém, alguns autores citam que este elemento geralmente não proporciona bons resultados de absorção quando aplicado via foliar, independentemente da formulação (Cabrita, 1993; Quaggio et al., 2003).

TABELA 3 - Teores de ferro e cálcio no sistema radicular de mudas de limoeiro 'Cravo' submetido a diferentes pulverizações com soluções contendo micronutrientes e extrato pirolenhoso em diferentes concentrações, aos 160 DAP. Jaboticabal, UNESP, 2003.

\begin{tabular}{|c|c|c|c|c|}
\hline TRATAMENTOS & \multicolumn{2}{|c|}{$\mathrm{Ca}\left(\mathrm{g} \mathrm{kg}^{-1}\right)$} & \multicolumn{2}{|c|}{$\mathrm{Fe}\left(\mathrm{mg} \mathrm{kg}^{-1}\right)$} \\
\hline Testemunha & 3,77 & $\mathrm{~b}$ & 560,25 & $\mathrm{a}$ \\
\hline Micro & 4,15 & $\mathrm{~b}$ & 554,25 & $\mathrm{a}$ \\
\hline Micro + EP $1 \mathrm{~cm}^{-3} \mathrm{dm}^{-3}$ & 5,27 & $\mathrm{a}$ & 378,25 & $\mathrm{~b}$ \\
\hline Micro + EP $2 \mathrm{~cm}^{-3} \mathrm{dm}^{-3}$ & 5,22 & $\mathrm{a}$ & 362,75 & $\mathrm{~b}$ \\
\hline Micro + EP $5 \mathrm{~cm}^{-3} \mathrm{dm}^{-3}$ & 5,37 & $\mathrm{a}$ & 288,25 & $\mathrm{~b}$ \\
\hline Micro + EP $10 \mathrm{~cm}^{-3} \mathrm{dm}^{-3}$ & 5,52 & $\mathrm{a}$ & 352,50 & $\mathrm{~b}$ \\
\hline DMS (Tukey) & \multicolumn{2}{|c|}{0,63} & \multicolumn{2}{|c|}{131,35} \\
\hline $\mathrm{CV}(\%)$ & \multicolumn{2}{|c|}{5,62} & \multicolumn{2}{|c|}{13,73} \\
\hline
\end{tabular}

Médias seguidas de mesma letra não diferem entre si, pelo teste de Tukey, a 5\% de probabilidade 
TABELA 4 - Teor de micronutrientes na parte aérea de limoeiro 'Cravo'submetido a pulverizações com soluções contendo micronutrientes e EP em diferentes concentrações, aos 160 DAP. Jaboticabal, UNESP, 2003.

\begin{tabular}{|c|c|c|c|c|c|}
\hline \multirow{2}{*}{ TRATAMENTOS } & $\mathrm{B}$ & $\mathrm{Cu}$ & $\mathrm{Fe}$ & $\mathrm{Mn}$ & $\mathrm{Zn}$ \\
\hline & \multicolumn{5}{|c|}{$\left(\mathrm{mg} \mathrm{kg}^{-1}\right)$} \\
\hline Testemunha & $108,00^{\mathrm{ab}}$ & $1,50 \mathrm{~b}$ & $79,00 \quad b$ & $13,75^{\mathrm{c}}$ & $11,50 \mathrm{c}$ \\
\hline Micro & $111,00 \mathrm{ab}$ & $3,25 b$ & $101,50 \mathrm{a}$ & $15,50 \mathrm{bc}$ & $15,50 \mathrm{ab}$ \\
\hline Micro + EP $1 \mathrm{~cm}^{-3} \mathrm{dm}^{-3}$ & $116,50 \mathrm{a}$ & $3,50 \mathrm{~b}$ & $96,25 \mathrm{a}$ & $16,25 b c$ & $15,00 \mathrm{~b}$ \\
\hline Micro + EP $2 \mathrm{~cm}^{-3} \mathrm{dm}^{-3}$ & $115,25 \mathrm{ab}$ & $4,25 \mathrm{ab}$ & $99,50 \mathrm{a}$ & $16,50 \mathrm{ab}$ & $16,75 \mathrm{ab}$ \\
\hline Micro + EP $5 \mathrm{~cm}^{-3} \mathrm{dm}^{-3}$ & $105,25 \mathrm{ab}$ & $4,25 \mathrm{ab}$ & $103,25 \mathrm{a}$ & $17,50 \mathrm{ab}$ & $15,75 \mathrm{ab}$ \\
\hline Micro + EP $10 \mathrm{~cm}^{-3} \mathrm{dm}^{-3}$ & $102,25 b$ & $7,00 \mathrm{a}$ & $108,00 \mathrm{a}$ & $19,00 \mathrm{a}$ & $17,75 \mathrm{a}$ \\
\hline $\mathrm{CV}(\%)$ & 5,24 & 31,14 & 6,39 & 6,67 & 7,08 \\
\hline
\end{tabular}

Médias seguidas de mesma letra não diferem entre si, pelo teste de Tukey, a 5\% de probabilidade.

$\mathrm{EP}=$ extrato pirolenhoso.

Trabalho desenvolvido por Santos et al. (1999), com a pulverização foliar de diversas formulações comercias (quelatos e sais) contendo B, Mn e Zn, em plantas de laranjeira 'Pêra' (C. sinensis Osbeck), enxertadas sobre limoeiro 'Cravo' $(C$. limonia Osbeck) e com dois anos de idade, não revelou diferenças significativas nos teores foliares de B, mesmo variando a concentração do elemento nas soluções. Silva (1996) avaliou o desempenho da aplicação foliar, em plantas de laranjeira 'Pêra' enxertadas sobre limoeiro 'Cravo' com seis anos de idade, com soluções contendo B (ácido bórico) em duas concentrações (85 e $50 \mathrm{mg} \mathrm{dm}^{-3} \mathrm{~B}$ ) e também não verificou aumento do teor do nutriente nas folhas colhidas 30 e 60 dias após a aplicação.

A solução de micronutrientes contendo $10 \mathrm{~cm}^{3} \mathrm{dm}^{-3}$ de EP induziu um aumento de $78 \%$ nos teores de cobre, se comparado à testemunha absoluta ( $\mathrm{T} 0$ = água sem nutrientes), e 54\% se comparado à solução de micronutrientes dissolvido em água (tratamento T1). Embora o extrato pirolenhoso possua $20,8 \mathrm{mg} \mathrm{dm}^{-3} \mathrm{de} \mathrm{Cu}$ em sua composição, mesmo na solução mais concentrada $\left(10 \mathrm{~cm}^{3} \mathrm{dm}^{-3}\right)$, a diluição baixa tal contribuição para valores desprezíveis frente ao adicionado através sulfato de $\mathrm{Cu}$, o que ficou constatado nos resultados da análise das soluções usadas no presente trabalho (Tabela 1). Tais resultados sugerem que a presença do extrato pirolenhoso na solução de micronutrientes foi o que proporcionou aumento da concentração de $\mathrm{Cu}$ na parte aérea das plantas de limoeiro 'Cravo'.

As deficiências de $\mathrm{Cu}$ são comumente notadas em viveiros de mudas cítricas, e Boaventura (2003) relatou maior exigência de cobre para mudas cítricas cultivadas em substrato orgânico, se comparada a plantas adultas. Nesse contexto, o EP poderia ser utilizado como um possível aditivo da calda de pulverização para auxiliar na absorção de $\mathrm{Cu}$ neste sistema de produção.

Para o Fe, foi constatado que a pulverização da solução contendo $250 \mathrm{mg} \mathrm{dm}^{-3}$ do elemento em água (T1) aumentou em aproximadamente $23 \%$ seu teor na parte aérea em relação à testemunha (T0), mas os tratamentos contendo micronutrientes, com ou sem extrato pirolenhoso, não diferiram entre si (Tabela 4). Todavia, vale mencionar que o teor de $\mathrm{Fe}$ aparentemente teve pequenos incrementos com o aumento da concentração de EP na calda.

No caso do Mn, observou-se que a pulverização dos micronutrientes dissolvidos apenas em água (T1) não alterou os teores do elemento na parte aérea, em comparação ao teor das plantastestemunha (T0). No entanto, foi constatado que a pulverização de solução contendo 2, 5 e $10 \mathrm{~cm}^{3} \mathrm{dm}^{-3}$ de EP aumentou em 18; 22 e 26\%, respectivamente, o teor de $\mathrm{Mn}$ na parte aérea, em relação à testemunha (T0), demonstrando que a adição do EP na calda de pulverização induziu aumentos significativos no teor de Mn, na parte aérea de plantas de limoeiro 'Cravo'.

De acordo com Boaretto et al. (2003), as folhas de citros têm capacidade limitada para absorver o Mn nelas depositado, e a quantidade absorvida é dependente da fonte utilizada. Os mesmos autores confirmaram que o Mn é mais absorvido na forma de cloreto, seguido pelo sulfato e, por último, pelo quelato (Mn-EDTA). Sabe-se, também, que o aumento da acidez não favorece a formação de quelatos de Mn (Basset et al., 1981), nem a absorção de cátions (Malavolta et al.,1997). Em função disso, pode-se inferir que o efeito estimulante do EP sobre a absorção de Mn não esteja vinculado à possível capacidade quelante do produto.

Os teores de zinco na parte aérea foram aumentados em cerca de $25 \%$ pela pulverização das soluções contendo o elemento (Tabela 4), porém não se observou variação consistente do teor de Zn na planta com o aumento do teor de EP na solução. Observou-se que a solução com EP a $1 \mathrm{~cm}^{3} \mathrm{dm}^{-3}$ proporcionou um teor de $\mathrm{Zn}$ menor que a solução sem EP, enquanto a de EP a $10 \mathrm{~cm}^{3} \mathrm{dm}^{-3}$ induziu um teor maior, de modo que a diferença entre elas foi significativa. Assim como se observou para $\mathrm{Fe}$, o extrato pirolenhoso não afetou a absorção do íon $\mathrm{Zn}$, pois todas as soluções contendo micronutrientes induziram aumentos semelhantes dos teores do nutriente na parte aérea, se comparada ao tratamento-testemunha (T0).

$\mathrm{O}$ aumento do teor de $\mathrm{Cu}$ e $\mathrm{Mn}$ na parte aérea das mudas de limoeiro 'Cravo' verificado na presente pesquisa indica que o EP, na concentração de $10 \mathrm{~cm}^{3} \mathrm{dm}^{-3}$, poderia ser usado na calda de pulverização da parte aérea das mudas quando se pretende aumentar a eficiência da aplicação destes elementos via foliar, visto que estes micronutrientes são aqueles que mais comumente se encontram em deficiência nas mudas e pomares adultos.

\section{CONCLUSÕES}

1. O extrato pirolenhoso destilado, adicionado à solução de micronutrientes e pulverizado na parte aérea a $1 ; 2 ; 5$ e $10 \mathrm{~cm}^{3} \mathrm{dm}^{-3}$ não interferiu na concentração foliar de B, Fe e $\mathrm{Zn}$ em mudas de limoeiro 'Cravo'.

2. A adição de extrato pirolenhoso a $10 \mathrm{~cm}^{3} \mathrm{dm}^{-3}$ em solução contendo micronutrientes aumentou a concentração foliar de $\mathrm{Cu}$ e $\mathrm{Mn}$ na parte aérea de mudas do limoeiro 'Cravo'.

3. A aplicação foliar de soluções de $\operatorname{EP}\left(1 \mathrm{a} 10 \mathrm{~cm}^{3} \mathrm{dm}^{-3}\right)+$ micronutrientes diminuiu a concentração foliar de $\mathrm{Fe}$ e aumentou a de $\mathrm{Ca}$ no sistema radicular de mudas de limoeiro 'Cravo'.

\section{REFERÊNCIAS}

BASSET, J.; DENNEY, R.C.;JEFFERY, G.H.; MENDHAM, J. Vogel: análise inorgânica quantitativa. 4.ed. Rio de Janeiro: Guanabara dois, 1981. 690p.

BIOCARBO INDÚSTRIA E COMÉRCIO. Curso prático de agricultura orgânica. Itabirito, 2003. 15p. (Apostila complementar Região do Irecê-BA).

BOARETTO, A.E.; ROSOLEM, C.A. Adubação foliar: Conceituação em prática. In: BOARETTO, A.E.; ROSOLEM, C.A. Adubação foliar. 2.ed. Campinas: Fundação Cargill, 1989. v. 2, p. 301-320.

BOARETTO, A.E.; MURAOKA, T.; BOARETTO, R.M. Absorção e translocação de Mn, Zn e B Aplicados via foliar em Citros. Laranja, 
Cordeirópolis, v. 24, n. 1,p. 177-197, 2003.

BOAVENTURA, P.S.R. Demanda por nutrientes de porta-enxertos e mudas cítricas produzidas em substrato em ambiente protegido. 2003. 61f. Dissertação (Mestrado em Agronomia) - Instituto Agronômico de Campinas, Campinas, 2003.

CABRITA, J.R.M. Aplicação de boro, manganês e zinco em citros vias solo e foliar, 1993. 47f. Dissertação (Mestrado em Agronomia) Faculdade de Ciências Agrárias e Veterinárias, Universidade Estadual Paulista, Julio de Mesquita Filho, Jaboticabal, 1993.

CAETANO, A.A. Estudo da eficiência de várias fontes dos micronutrientes, zinco, manganês e boro aplicados em pulverizações na laranjeira 'Valência'(Citrus sinensis (L.) Osbeck). 1982. 46f. Dissertação (Mestrado em Agronomia). Escola Superior de Agricultura Luiz de Queiroz, Universidade de São Paulo, Piracicaba, 1982.

CAMARGO, P.N.; SILVA, O. Manual de adubação foliar. São Paulo:La Libéria / Herba: São Paulo, 1975. 258p.

CARVALHO, S.A. Regulamentação atual da Agência de Defesa Agropecuária para produção, estocagem, comércio, transporte e plantio de mudas cítricas no Estado de São Paulo. Laranja, Cordeirópolis, v. 24, n. 1, p. 199-239, 2003.

DECHEN, A.R.; HAAG, H.P.; CARMELLO, Q.A.C. Mecanismos de absorção de nutrientes e translocação de mucronutrientes. In: MICRONUTRIENTES NAAGRICULTURA. Piracicaba: POTAFOS/ CNPq., 1991.p. 79-98,

ESECHIE, H.A.; DHALIWAL, G.S.; ARORA, R.; RANDHAWA, N.S.; DHAWAN, A.K. Assessment of pyroligneous liquid as a potential organic fertilizer. In: ECOLOGICAL AGRICULTURE AND SUSTAINABLE DEVELOPMENT, 1997, Chandigarh, India. Proceedings... Chandigarh: Center for Research in Rural and Industrial Development, 1998. v.1, p. 591-595.

MALAVOLTA, E.; VITTI, G.C.; OLIVEIRA, S.A.DE. Avaliação do estado nutricional das plantas; princípios e aplicações. 2.ed. Piracicaba: Associação Brasileira para Pesquisa da Potassa e do Fosfato, 1997. 201p.

MIYASAKA, S.; OHKAWARA, T.; NAGAI, K.; YAZAKI, H.; SAKITA, M.N. Técnicas de produção e uso do fino de carvão e licor pirolenhoso In: ENCONTRO DE PROCESSOS DE PROTEÇÃO DE PLANTAS: Controle ecológico de pragas e doenças, 1., 2001, Botucatu. Resumos... p.161-176.

PEDRAS, J.F.; RODRIGUES, J.D.; RODRIGUES, S.D. Absorção de íons via foliar. In: BOARETTO, A.E.; ROSOLEM, C.A. Adubação foliar. 2.ed. Campinas: Fundação Cargill, 1989. v. 2, p. 301-320.
PONCHIO, C.O.; BALLIO, L.A.C. Fontes de enxofre e micronutrientes para a agricultura Brasileira. In: ENXOFRE E MICRONUTRIENTES NAAGRICULTURA BRASILEIRA, 17., 1988, Londrina. Anais... Londrina: EMBRAPA-CNPSo/IAPAR/SBCS, 1988. p. 265-276.

QUAGGIO, J.A.; MATTOS JUNIOR, D.; CANTARELLA, H.; TANK JUNIOR,A. Fertilização com boro e zinco no solo em complementação à aplicação via foliar em laranjeira 'Pêra'. Pesquisa agropecuária brasileira, Brasília/DF, v.38 n.5. 2003.

QUAGGIO, J. A.; RAIJ, B. van; PIZAJUNIOR, C. T. Frutíferas. In: RAIJ, B. van; CANTARELLA, H.; QUAGGIO, J. A.; FURLANI, A. M. C. (Ed.). Recomendações de adubação e calagem para o Estado de São Paulo. 2. ed. Campinas: Instituto Agronômico, 1997. p. 121-153.

SANTOS, C.H.; DUARTE FILHO, J.; MODESTO, C.J.; GRASSI FILHO, H.; FERREIRA, G. Adubos foliares quelatizados e sais na absorção de boro, manganês e zinco em laranjeira 'Pera'. Scientia Agricola, Piracicaba, v. 56, n. 4, p. 999-1004, 1999.

SHIBAYAMA, H.; MASHIMA, K.; MITSUTOMI, M.; ARIMA, S. Effects of application of pyroligneous acid solution produced in Karatsu city on growth and free sugar contents of storage roots of sweet potatoes. Marine and Higland Bioscience Center Report, Phukel, v. 7, p. 15-23. 1998.

SILVA, M.M. Adubação dos teores foliares de micronutrientes em citros em função da aplicação de fungicidas, sais e quelatizaos. 1996. 58f. Dissertação (Mestrado em Agronomia) - Escola Superior de Agricultura Luiz de Queiroz, Universidade de São Paulo, Piracicaba, 1996.

SONNEVELD, C.; ENDE, J.V.D.; DIJK, P.A.V. Analysis of growing media by means of a 1:1,5 volume extract. Communications in Soil Science an Plant Analysis, v. 5, n. 3, p.183-202, 1974.

TEDESCO, M.J.; VOLWEISS, S.J.; BOHNEN, H. Análise de solos, plantas e outros materiais. Porto Alegre: FA/Universidade Federal do Rio Grande do Sul, 1995. 188p. (Boletim técnico, 5).

TIRITAN, S.T. Adubação foliar de micronutrientes em citros. 1996. 64f. Dissertação (Mestrado em Agronomia) - Escola Superior de Agricultura Luiz de Queiroz, Universidade de São Paulo, Piracicaba, 1996.

TSUZUKI, E.; MORIMITSU, T.; MATSUI, T. Effect of chemical compounds in pyroligneous acid on root growth in rice plant. Japan Journal Crop Science, Tokyo, v. 66, n. 4, p. 15-16, 2000.

WALLACE, A. Introduction: Metal Chelates in Agriculture. In: SYMPOSIUM ON THE USE OF METAL CHELATES IN PLANT NUTRITION, 1., 1956, Palo Alto/CA. Proceedings... Palo Alto/ CA,1956. p.4-23. 